\title{
A QUALIDADE DA CERTIFICAÇÃO MÉDICA DA CAUSA DE MORTE EM SÃO PAULO, BRASIL *
}

\author{
Luiz Augusto Marcondes FONSECA **
}

Ruy LAURENTI **

RSPU-B/200

\begin{abstract}
FonsecA, L. A. M. \& LAURENTI, R. - Qualidade da certificação médica da causa de morte em São Paulo, Brasil. Rev. Saúde públ., S. Paulo, 8: $21-9,1974$.

Resumo: Foi feita análise dos atestados de bbito, do preenchimento dos seus itens e da declaração da causa básica da morte. Foi estudada uma amostra de óbitos ocorridos em hospitais e pronto socorros no periodo de 1.0 de março de 1971 a 29 de fevereiro de 1972. Foram colhidas informaçōes adicionais, para cada caso, a partir dos prontuários médicos, o que permitiu conhecer a verdadeira causa básica da morte e comparar com o que foi declarado no atestado. Verificou-se que somente $4,0 \%$ dos atestados apresentaram informações sobre o intervalo de tempo entre o inicio da doenca e a morte e sobre o exame complementar que confirmou o diagnóstico, sendo que $65,5 \%$ não apresentaram ambas as informações. $O$ item mais negligenciado foi o referente ao intervalo de tempo, pois em 93,5\% dos atestados este item não se encontrava preenchido; $68,0 \%$ dos atestados não tinha preenchido o item sobre exame complementar que confirmou o diagnóstico. Quanto a declaração da verdadeira causa básica foi verificado que $31,4 \%$ dos atestados não a apresentavam registrada $e 6,5 \%$, ainda que a tivessem registrada, não era selecionada como a causa primária da morte para finalidades de estatística de mortalidade. Conclui-se que não é boa a qualidade dos atestados de óbitos na cidade de São Paulo, e comparando os resultados com os de estudo semelhante feito em 1962/1963, verifica-se que não houve melhora na certificação médica da causa de morte nos últimos 10 anos.
\end{abstract}

Unitermos: Atestados de bbito (São Paulo, Brasil)*; Estatistica vital*; Mortalidade*.

\section{N T R O D U C A O}

A fonte de dados das estatísticas de mortalidade é o atestado de óbito, razão pela qual se torna desnecessário salientar a importância do seu correto preenchimento. 0 atual modelo do atestado de óbito - modelo internacional - foi pro- posto em 1948 e é usado por, praticamente, todos os países.

Segundo recomendações da OMS, as causas de morte a serem registradas nos atestados de óbito são "todas as doenças, estados mórbidos e lesóes que produziram

* Trabalho realizado com auxílio financeiro da FAPESP (Med. 72/725).

* Do Departamento de Epidemiologia da Faculdade de Saúde Pública da USP - Av. Dr. Arnaldo, 715 -- São Paulo, SP - Brasil. 
FONSECA, L. A. M. \& LAURENTI, R. - A qualidade da certificação médica da causa de morte em São Paulo, Brasil. Rev. Saúde públ., S. Paulo, 8: 21-9, 1974

a morte ou que contribuiram para ela, e as circunstâncias do acidente ou da violência que produziu essas lesões". Para finalidades estatísticas, a classificação da causa de morte é simples quando só uma causa é mencionada; quando são mencionadas várias afecções uma delas é selecionada e é a chamada "causa-básica". Esta é definida como: (a) doença ou lesão que iniciou a cadeia de acontecimentos patológicos que conduziram diretamente à morte, ou (b) circunstâncias do acidente ou violência que produziram a lesão fatal" 5

Para assegurar uma aplicação uniforme daquele princípio é que foi proposto - "Modelo Internacional de Atestado Médico", recomendado pela Assembléia Mundial de Saúde em $1948^{5}$, o qual foi concebido de maneira a facilitar ao médico a declaração da causa básica segundo a definição da OMS.

$\mathrm{O}$ atestado de óbito é constituído das partes I e II e dos ítens referentes ao intervalo de tempo que duraram as doenças mencionadas e aos exames complementares que confirmaram o diagnóstico. $\mathrm{Na}$ parte I deverá ser registrada a causa básica e em sequêencia as complicações, se houver. A parte II é reservada para o registro das chamadas causas contribuintes, quando houver.

Tem sido verificado que as estatísticas de mortalidade nem sempre são inteiramente fidedignas, o que se deve, em grande parte, ao fato de os atestados de óbitos não serem corretamente preenchidos. Na cidade de São Paulo, para o periodo 1962/1963 foi feita uma avaliação da qualidade do atestado de óbito ${ }^{\circ}$, tendo sido verificado que apenas $5,5 \%$ dos atestados tinham todos os itens preenchidos e estavam com seqüência lógica na parte I; aquela proporção se elevou a $8,2 \%$ se não se levasse em conta a sequiência da parte I. Por outro lado, verificou-se que somente $67,6 \%$ dos atestados tinham escrita, em qualquer parte, a verdadeira causa básica.
Vários trabalhos têm sido publicados avaliando qualitativamente as informaçôes existentes nos atestados de óbito e, entre outros, podemos citar: JAMEs et al. ${ }^{3}$, Abramson et al. ${ }^{1}$, Puffer \& SerRANO ${ }^{8}$, MORIYAMA et al. ${ }^{7}$, BEADENKOPF et al. ${ }^{2}$.

Em 1971 e 1972 foi realizado no Departamento de Epidemiologia da Faculdade de Saúde Pública uma investigação sobre causas múltiplas de morte, quando foi estudada uma amostra de óbitos ocorridos em hospitais da cidade de São Paulo, coletando-se para cada caso informações adicionais existentes nos prontuários médicos, para permitir melhor avaliação da causa básica e tornar possível a análise mais completa das causas múltiplas de morte ${ }^{4}$. Aproveitamos o material existente - atestados de óbitos e informações adicionais - para uma análise qualitativa dos atestados de óbitos.

O B J E T I OS

Objetivamos neste trabalho o seguinte:

- análise do preenchimento dos atestados de óbito, levando-se em conta os itens existentes no mesmo (partes I e II, intervalo de tempo e exames que confirmaram o diagnóstico);

- verificação da proporção de atestados em que foi declarada a verdadeira causa básica.

\section{MATERIAL E METODOS}

O material utilizado foi o mesmo da "Investigação de Causas Múltiplas de Morte" ${ }^{\circ}$. Os atestados analisados foram aqueles referentes a óbitos ocorridos em hospitais ou pronto socorros e registrados no distrito de São Paulo, no período de $10^{\circ}$ de março 1971 a 29 fevereiro 1972. 0 número de óbitos, para o período, foi estimado em 51.000 sendo que, aproximadamente, $70 \%$ ocorriam em hospitais 
FONSECA, L. A. M. \& LALRENTI, R. - A qualidade da certificação médica da causa de morte em São Paulo, Brasil. Rev. Sailde públ., S. Paulo, 8: 21.9, 1974.

ou pronto socorros. Foi colhida uma amostra probabilística sistemática (um para cada 19 óbitos), tendo sido sorteados 1.895 atestados.

Para cada caso sorteado foram colhidas informações através de consultas aos prontuários existentes no hospital, anotando-se dados de história clínica, exames, cirurgia, etc. Essas informações permitiam conhecer a causa básica da morte. para cada caso, e comparar com o que foi declarado no atestado.

Quanto a análise do preenchimento dos atestados de óbitos, eles foram classificados como:

a) Completos: aqueles em que, além da parte I, também estavam preenchidos $o$ itens correspondentes ao intervalo entre o início do processo mórbido e o óbito e - referente ao exame complementar que confirmou o diagnóstico. A parte II não foi levada em consideração, pois em muitos casos não existem causas contribuintes; no entanto, foi verificada a proporção de atestados completos que trazem essa informação.

Os atestados completos foram considerados completos com sequiência, quando tinham sequência causal lógica na parte I, não importando houvesse sido ou não informada a verdadeira causa básica. Completos sem seqüência eram aqueles que não apresentavam sequência lógica na parte I, podendo da mesma maneira ter ou não informada a verdadeira causa básica.

b) Incompletos: assim foram considerados os atestados que não apresentaram preenchidos os itens: intervalo de tempo, exame que confirmou o diagnóstico, ou ambos; nesta categoria, os atestados também podiam ser divididos em dois grupos: com seqüência e sem seqüencia.

c) Atestados corretos: aqueles que apresentavam a mesma causa básica que a escolhida após conhecidas as informações adicionais através das consultas aos prontuários médicos. Quando a causa básica estava informada no atestado em sequência lógica, ele era considerado como correto no original. Quando esta causa não estava informada em seqüência lógica, porém após aplicação das "Regras de Seleção da Causa Básica de Morte" ela era selecionada, o atestado era considerado como correto após regras.

d) Atestados incorretos: foram assim considerados aqueles que não apresentavam declarada a verdadeira causa básica.

e) Foi considerado também um caso particular de atestado: aquele que apresentava declarada a verdadeira causa básica, porém colocada de tal maneira que mesmo aplicando as regras de seleção ela não era selecionada como tal. São atestados incorretos mas que tem escrita a verdadeira causa básica.

\section{RESLITADOS E DISCUSSAO}

Dos 1.895 atestados sorteados, 63 foram excluídos da análise por vários motivos. A análise refere-se então a 1.832 atestados.

Preenchimento do atestado de óbito: foram excluídos da análise 137 casos: isto ocorreu porque estes atestados foram fornecidos pelo Instituto Médico Legal (IML) que utiliza modelo diferente não apresentando as características do modelo internacional. A certificação usada pelo IML tem apenas lugar para registrar a causa ou causas de morte, sem ordem de seqüência. Além do mais, não apresenta os itens referentes a intervalo de tempo e a exames complementares que confirmaram o diagnóstico. 
FonseCA, L. A. M. \& LAURENTI, R. - A qualidade da certificação médica da causa de morte em São Paulo, Brasil. Rev. Saúde públ., S. Paulo, 8: 21-9, 1974.

TABELA 1

Atestados de óbitos segundo o preenchimento dos itens "Intervalo entre o início do processo mórbido e o óbito" (A) e "Exame complementar que confirmou o diagnóstico" (B)

\begin{tabular}{|c|c|c|c|c|c|c|}
\hline \multirow[b]{2}{*}{ (B) } & \multicolumn{2}{|c|}{ Sim } & \multicolumn{2}{|c|}{ Não } & \multicolumn{2}{|c|}{ Total } \\
\hline & n. ${ }^{\circ}$ & $\%$ & n.o & $\%$ & n.o & $\%$ \\
\hline Sim & 69 & 4,0 & 474 & 28,0 & 543 & 32,0 \\
\hline Não & 42 & 2,5 & 1110 & 65,5 & 1152 & 68,0 \\
\hline Tota1 & 111 & 6,5 & 1584 & 93,5 & 1695 & 100.0 \\
\hline
\end{tabular}

Os resultados mostram que, quanto ao preenchimento dos itens "A" e "B", a qualidade dos atestados não é boa. De fato, somente $4,0 \%$ dos atestados apresentaram ambos preenchidos e $65,5 \%$ não os tinham preenchidos. O preenchimento do item "intervalo entre o início do processo mórbito e o óbito" (A) foi mais negligenciado, pois $93,5 \%$ dos atestados não o apresentavam declarado. Quanto ao item "exame complementar que confirmou o diagnóstico" (B), 68,0\% dos atestados não continham essa informação.

No trabalho publicado por MiLANesi \& LAUREXTI ${ }^{6}$, onde foram analisados os mesmos aspectos para os óbitos ocorridos em 1962/1963, os atestados completos (itens A e B preenchidos) representaram $8,2 \%$. A comparação resulta desfavorável para a amostra do presente estudo, pois somente $4,0 \%$ foram considerados completos. Outro fato a destacar é que, no estudo feito anteriormente, a amostra de atestados englobava óbitos hospitalares ou não, enquanto que o atual só analisou os ocorridos em hospitais e pronto-socorros. Lma idéia que se poderia ter é de que esse fato contribuiria para uma melhor qualidade no preenchimento dos atestados, o que, como se vê pelos resultados não está ocorrendo.

A falta de preenchimento dos itens "A" $e$ " $\mathrm{B} "$ a rigor, não afeta as estatísticas de mortalidade. $O$ que se deve ter em mente, porém, é que o atestado é um formulário que deve ter todos os itens preenchidos. 0 fato de isto não acontecer levanos a concluir que não se presta a devida atenção nem se compreende a importância do atestado como fonte de informaçōes. Para a maioria dos médicos o atestado é apenas uma formalidade que serve para o sepultamento. Ignora-se, na maioria das vezes, que esse material é importante em saúde pública e em estudos epidemiológicos. Também é ignorado o fato de que o atestado de óbito pode ser usado em estudos clínicos do tipo "seguimento de casos". Para tal estudo interessa o preenchimento do item " $A$ ". No entanto, se se quiser realizar uma análise utilizando esse dado, entre nós isso será impossível pois apenas em $6,5 \%$ das vezes os atestados trazem informações sobre o intervalo de tempo decorrido entre o início da doença e a morte.

0 preenchimento do item relativo aos exames que confirmaram o diagnóstico (B) é importante pois serve como confirmação dos diagnósticos declarados. E claro que muitas vezes os diagnósticos são baseados somente em evidências clínicas. Nesses casos o médico deveria, da mesma maneira, informar isso, escrevendo "exame clínico". Na amostra analisada con- 
FONSECA, L. A. M. \& LAURENTI, R. - A qualidade da certificação médica da causa de morte em São Paulo, Brasil. Rev. Saúde públ., S. Paulo, 8: 21-9, 1974.

sideramos preenchido o item quando essa informação estava registrada.

A falta de preenchimento da parte II dos atestados não é elemento para julgar sobre a qualidade dos mesmos; na amostra foi verificado que em $78,6 \%$ das vezes não existia nenhuma informação registrada. Na análise feita em $1962 / 1963^{6}$, essa proporção foi $87,0 \%$, o que não nos permite dizer que os médicos estejam preenchendo mais o item II, pois poderiam estar existindo, atualmente, causas contribuintes, em maior número de vezes, do que há 10 anos. Por outro lado, no presente estudo, apenas foram considerados os óbitos ocorridos em hospitais, onde existem maiores facilidades para diagnosticar outras doenças concomitantes àquela que causou a morte.

Declaração da verdadeira causa básica nos atestados: Esse é o aspecto mais importante na análise qualitativa dos atestados de óbitos e o que realmente mede a fidedignidade das informações relativas à mortalidade por causa, sendo uma preocupação constante dos que trabalham com estatísticas vitais.

Para este tipo de análise consideramos quatro tipos de atestados, como foram definidos em Material e Métodos: corretos no original, corretos após regras, incorretos e incorretos mas que tem escrita a verdadeira causa básica.

TA B E A 2

Atestados de óbitos segundo a declaração da verdadeira causa básica

\begin{tabular}{l|c|c}
\hline \multicolumn{1}{c|}{ Atestados } & n.० & $\%$ \\
\hline \hline Corretos no original & 920 & 50,3 \\
Corretos apos regras & 216 & 11,8 \\
Incorretos & 576 & 31,4 \\
Incorretos mas que tem & & \\
escrita a verdadeira cau- & & \\
sa- básica & 120 & 6,5 \\
\hline$-\square$ & 1832 & 100,0 \\
\hline Total & & \\
\hline
\end{tabular}

Somente $50,3 \%$ dos atestados foram considerados corretos no original, representando aqueles em que os médicos declaram a verdadeira causa básica e de maneira adequada. Sob o ponto de vista de fidedignidade de estatística de mortalidade por causa, a esse valor devem ser acrescidos os $11,8 \%$ correspondentes aos atestados em que a causa básica verdadeira, embora declarada de maneira inadequada, é selecionada como causa da morte após a aplicação das regras de seleção.

Foi verificado que $31,4 \%$ dos atestados não apresentavam a verdadeira causa da morte declarada e que em $6,5 \%$ ela estava declarada porém não era selecionada como básica após aplicação das regras de seleção. Portanto, não há exatidão quanto a mortalidade por causa em $37,9 \%$ dos casos.

No estudo feito em $1962 / 1963^{6}$ foram considerados corretos os atestados que apresentavam a verdadeira causa básica, independente de ser anotada de maneira adequada e inclusive mesmo que não fosse selecionada como básica por aplicação de regras. Foi verificado que $67.6 \%$ possuiam essas características. Para comparar esse resultado com o atual é necessário, neste, scmar as categorias "corretos no original", "corretos após regras" e "incorretos mas que tem escrita a verdadeira causa básica" que representam $68,6 \%$. Como se vê, no período de 10 anos, em nada melhorou a qualidade dos atestados de óbitos na cidade de São Paulo. Mais uma vez chamamos a atenção para o fato de que no presente estudo somente foram analisados óbitos hospitalares, onde seria de se esperar uma melhor qualidade dos atestados.

Para um conjunto de atestados de óbito de menores de 5 anos de idade, de diferentes áreas das Américas, foi verificado que somente $52,5 \%$ apresentavam declarada a verdadeira causa básica; para a cidade de São Paulo, incluida nessa pesquisa, o valor observado foi de $50,2 \%^{8}$. sendo que foram estudados óbitos ocor- 
FONSECA, L. A. M. \& LAURENTI, R. - A qualidade da certificação médica da causa de morte em São Paulo, Brasil. Rev. Saúde pribl., S. Paulo, 8: 21-9, 1974.

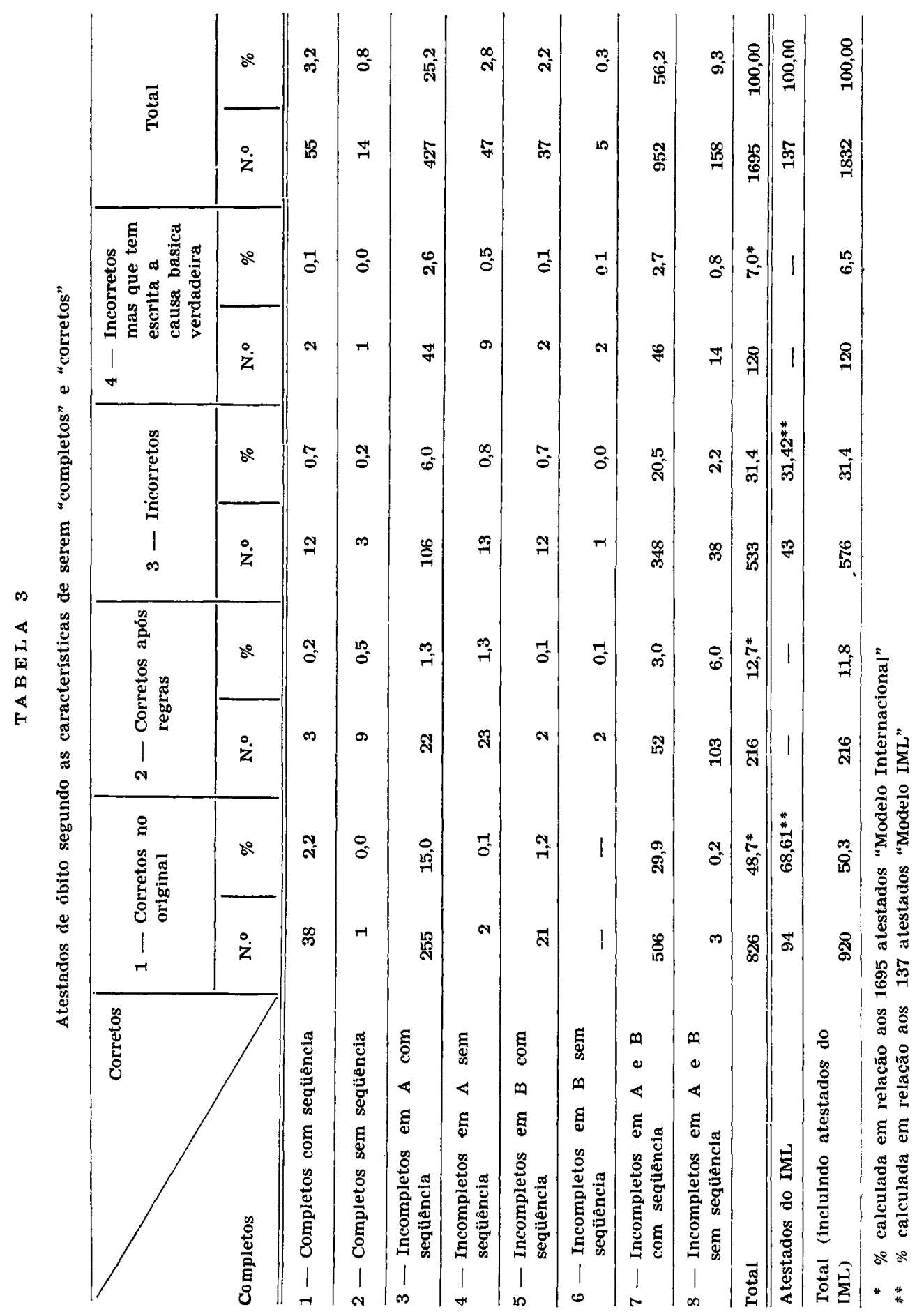


FONSECA, L. A. M. \& LAURENTI, R. - A qualidade da certificação médica da causa de morte em Săo Paulo, Brasil. Rev. Saúde públ., S. Paulo, 8: 21-9, 1974.

ridos ou não em hospitais, no período de junho 1968 a maio 1970 .

Uma apreciação melhor e mais completa sobre a qualidade dos atestados pode ser encontrada na Tabela 3 , onde os mesmos estão apresentados segundo o fato de serem completos (preenchimento dos itens) e corretos (verdadeira causa básica declarada).

$O$ atestado de óbito ideal seria aquele "completo com seqüência" e "correto no original" o que foi observado em apenas $2,2 \%$ das vezes. O tipo de atestado mais deficiente é o "incorreto" e "incompleto em A e B sem seqüência", que coincidentemente também foi observado em $2,2 \%$ das vezes. Observados em maior freqüência, aproximadamente $30 \%$, foram aqueles "incompletos em A e B com seqüência" e "corretos no original".

$\mathrm{Um}$ aspecto que precisa ser salientado é que aproximadamente $85 \%$ dos atestados têm seqüência, independente de serem corretos $\mathrm{e} / \mathrm{ou}$ completos. Tal fato indica que os médicos ao preencherem os atestados, na grande maioria das vezes, registram as causas segundo uma ordem lógica como se requer no modelo internacional. Aparentemente, não há dificuldades quanto a maneira de se registrar as causas no atestado, parecendo mesmo que o grande problema é o desconhecimento do conceito de causa básica. De fato isso é sugerido pela grande proporção de atestados incorretos.

Os atestados expedidos pelo IML somente foram analisados quanto à caracteristica de serem ou não corretos, verificandose que $68,6 \%$ eram corretos no original. Foi observado que nesses atestados nunca é registrada a verdadeira causa básica da morte, a qual, no caso de mortes violentas, são "as circunstâncias do acidente ou violência", de acordo com a definição da OMS. Assim sendo, segundo a metodologia de análise adotada, todos esses ates- tados seriam incorretos. Ocorre, porém, que o órgão encarregado de elaborar as estatisticas de mortalidade *, ao trabalhar com esses atestados faz uma verificação nos laudos de autópsia correspondentes, assinalando as circunstâncias da causa acidental ou violência (causa externa), codificando-a como causa básica. Como o interesse do presente estudo foi, entre outros, avaliar a qualidade dos atestados tendo-se em vista a fidedignidade estatística, foram considerados corretos os atestados nos quais havia concordância entre a causa básica assinalada a posteriori pelo Departamento de Estatística * e aquela que havia sido escolhida através da leitura dos prontuários médicos existentes nos hospitais.

Os resultados obtidos mostram, mais uma vez, que não é satisfatório o preenchimento dos atestados de óbitos. 0 que mais afeta as estatísticas de mortalidade é a falta de precisão na declaração da causa básica pois, como foi visto, em $37,9 \%$ das vezes não houve concordância entre o que o médica registrou no atestado e aquilo que realmente ocorreu.

A melhoria das estatísticas de mortalidade, quanto a fidedignidade, somente será conseguida quando houver um preenchimento satisfatório dos atestados de óbito, principalmente no que se refere a declaração correta das causas que levaram à morte. Além deste aspecto, para alguns tipos de análises é necessário, também, que os atestados sejam preenchidos em todos os seus itens.

\section{CONCLUSOES}

A análise de uma amostra de atestados referentes a óbitos ocorridos em hospitais ou pronto socorros da cidade de São Paulo, no período de $10^{\circ}$ de março 1971 a 29 de fevereiro 1972 mostrou que o preenchimento dos mesmos não é satisfató-

- Departamento de Estatística da Secretarla de Economia e Planejamento do Estado de Săo Paulo. 
FonseCA, L. A. M. \& LAURENTI, R. - A qualidade da certificação médica da causa de morte em Săo Paulo, Brasil. Rev. Saúde pübl., S. Paulo, 8: 21-9, 1974.

rio. Assim, somente 4,0\% dosatestados tinham preenchido os itens referentes ao intervalo de tempo entre o início do processo mórbido e a morte e sobre exames complementares que confirmaram o diagnóstico. 0 item mais negligenciado foi aquele referente ao intervalo de tempo sendo que $93,5 \%$ dos atestados não o apresentavam preenchido; $68,0 \%$ não apresentavam informaçôes sobre o exame complementar que confirmou o diagnóstico.
Quanto à declaração da verdadeira causa básica da morte, a comparação dos atestados com informações adicionais obtidas através dos prontuários médicos mostrou que em $37,9 \%$ dos casos não havia concordância.

Os resultados atuais, comparados com estudo semelhante feito em 1962/1963, mostraram que nesses 10 anos não houve melhoria no preenchimento dos atestados de óbito na cidade de São Paulo.

RSPU-B/200

FONSECA, L. A. M. \& LAURENTI, R. - [The quality of medical certification for the cause of death in S. Paulo, Brazil.] Rev. Saúde públ., S. Paulo, 8: $21-9,1974$.

SUMMARY: It was made an analysis of the medical certifications of death; it was analysed the fulfilling of the items of the certifications and the declaration of the underlying cause of death. A study had been made on a sample of the deaths occurred in hospitals in the period from March 1971 to February 1972. Additional information about each case was obtained through medical records; so, it was possible to know the real underlying cause of death and compare this one with that stated in the original certification. It was observed that, due to the lack of fulfilment of the corresponding items, only 4.0 per cent of the certifications brought together information about the time break between the onset of the disease and death and information about the laboratory data that confirmed the diagnosis. It was also observed that 65.5 per cent didn't show any information about the referred items. The most neglected item was that related to the time interval: 93.5 per cent of the certifications didn't carry it; 68.0 per cent didn't show informations about the laboratory data that confirmed the diagnosis; 31.4 per cent of the certifications didn't carry any statement about the real underlying cause of death; 6.5 per cent, though carrying it, were fulfilled in such an inadequate way that it became impossible to select the underlying cause of death for statistical purposes. It was concluded that the quality of the medical certifications of death of the city of $\mathbf{S}$. Paulo is not satisfactory; comparing the results of this research with those of a similar one made in 1962/1963 it was verified that no improvement was observed in the last 10 years.

Uniterms: Death certificate (S. Paulo, Brazil) *; Vital statistics *; Mortality*.

\section{REFERENCIAS BIBLIOGRAFICAS}

1. ABRAMSON, J. H. et al. - Death certificate data as an indication of certain common diseases at death. $J$. chron. Dis., 24: 417-31, 1971.

2. BEADENKOPF, W. G. et al. - An assessment of certain medical aspects of death certificate data for epidemio- logic study of artherioesclerotic heart diseases. J. chron. Dis., 16: 249-62, 1962.

3. JAMES, G. et al. - Accuracy of cause of death statements on death cert1ficates. Publ, Hlth. Rep., ro: 39-51, 1955. 
FONSECA, L. A. M. \& LAURENTI, R. - A qualidade da certificaçáo médica da causa de morte em São Paulo, Brasil. Rev. Saúde públ., S. Paulo, 8: 21-9, 1974.

4. LAURENTI, R. - Causas múltiplas de morte. São Paulo, 1973. [Tese de Livre-docência - Faculdade de saúde Pública da USP].

5. MANUAL de classificação estatística internacional de doença, lesões e causas de óblto: $8 .^{\text {a }}$ revisão. Washington, D.C. Organização Panamericana de Saúde, 1969 (Publicação Científica, 190).

6. MILANESI, M. L. \& LAURENTI, R. O estudo interamericano de mortalidade em Săo Paulo. I - Estado atua] da certificação médica da causa de óbito no distrito da Capital. Rev. Ass. Méd. bras. 10: 111-16, 1964.
7. MORIYAMA, J. M. et al. - Evaluation of diagnostic information supporting medical certification of deaths from cardiovascular disease. In: HAENSZEL, W. ed. - Epidemiological approaches to the study of cancer and chronic diseases. Bethesda, Md., National Cancer Institute, 1966. p. 405-19.

8. PUFFER, R. R. \& SERRANO, C. V. Patterns of mortality in childhood, Washington, D.C., Pan American Health Organization, 1973 (Scientific Publication, 262).

Recebido para publicą̧̃o em 19-12-1973.

Aprovado para publicação em 21-1-1974. 